\title{
A PROOF OF SOME SCHÜTZENBERGER-TYPE RESULTS FOR EULERIAN PATHS AND CIRCUITS ON DIGRAPHS
}

\author{
BYOUNG-SONG CHWE \\ Department of Mathematics \\ University of Alabama \\ Tuscaloosa. Alabama 35486 \\ (Received December 30, 1992 and in revised form October 18, 1993)
}

ABSTRACT. This paper shows that the number of even Eulerian paths equals the number of odd Eulerian paths when the number of arcs is at least twice the number of vertices of a digraph.

KEY WORDS AND PHRASES. Digraph, Eulerian paths, odd permutation, even permutation. 1992 AMS SUBJECT CLASSIFICATION CODE. 05C30.

\section{INTRODUCTION AND CONVENTIONS.}

This paper shows that in a digraph of order $n$ with $m$ arcs that satisfies $m \geq 2 n$, the number of even Eulerian paths equals the number of odd Eulerian paths. This result generalizes Schützenberger's theorem (see [1] and [2]), which says that in a digraph of order $n$ with $m$ arcs that satisfies $m \geq 2 n+1$, the number of even Eulerian circuits equals the number of odd Eulcrian circuits. The proof is also perhaps more intuitive, not depending on too much terminology or disparate graph theory results.

In this paper, a digraph is defined by a sequence of arcs, where an arc is indicated by an ordered pair such as $(a, b)$, and multiple arcs and loops are allowed. Those letters which appear in describing the arcs in the sequence defining the graph are therefore considered as vertices or points. Note that with this definition, there can be no isolated vertices. If $D$ is a sequence of arcs, its length is denoted by $|D|$. If $V D$ denotes the set of vertices which appear in $D$, then $|V D|$ denotes its cardinality.

Two digraphs $D_{1}$ and $D_{2}$ are considered to be the same, under appropriate relabeling of vertices, when $D_{2}$, as a sequence of arcs, is a permutation of the sequence of arcs which define $D_{1}$. We write a sequence such as $((a, b),(c, d),(d, f))=D$ in the form $D=(a, b) \cdot(c, d) \cdot(d, f)$. For example, the digraph whose diagram is

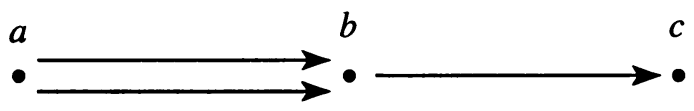

can be expressed as $D_{1}=(a, b) \cdot(b, c) \cdot(a, b)$ or $D_{2}=(a, b) \cdot(a, b) \cdot(b, c)$ with $D_{1}=D_{2}$ according to our definition. Also, $|D|=3$ and $|V D|=3$.

Unlike the usual way, we define a path of $D$ as a subsequence of a permutation of the sequence describing the digraph $D$. If a path $\left(a_{1}, b_{1}\right) \cdot\left(a_{2}, b_{2}\right) \cdot \cdots \cdot\left(a_{n}, b_{n}\right)$ is a permutation of $D$ and has the additional property that $b_{1}=a_{2}, b_{2}=a_{3}, \ldots, b_{n-1}=a_{n}$, then it is an Eulerian path of $D$. The added condition $b_{n}=a_{1}$ identifies an Eulerian circuit of $D$. A path $(a, b) \cdot(c, d) \cdot \cdots \cdot(e, f)$ is said to start from $a$ and end at $f$; the vertex $a$ is the starting point and the vertex $f$ is the end point. If $\alpha$ and $\beta$ are paths of $D$, then the sequence obtained by putting $\beta$ after $\alpha$ is written $\alpha \beta$ provided that the resulting sequence is also a path of $D$. 
Next, we denote by $\bigwedge_{x} D$ the set of all Eulerian paths of the digraph $D$ which start from the point $x$. If the starting point is fixed but unimportant we write $\wedge D$. Also, when $\alpha \in \Lambda_{x} D$. If we consider $\alpha$ as a digraph, then $\bigwedge_{x} \alpha=\bigwedge_{x} D$.

If $\alpha$ and $\beta$ are two paths and if there are two paths $\gamma$ and $\delta$. where either or both of $\gamma$ and $\delta$ can be empty, such that $\gamma \alpha \delta=\beta$, then $\alpha$ is a part of $\beta$ and we use the notation $\alpha \subseteq 3$ to inducate this fact. Hence, if an arc $(a, b)$ is a term in a path $\beta$, we write $(a, b) \subseteq \beta$. By $\beta-\alpha$ is meant the subsequence of $\beta$ obtained by climinating all terms of $\alpha$ one by one. Thus, if $\operatorname{arcs}$ in $\beta$ or $\alpha$ occur more than once the procedure may leave copies left over and the result may not be unique. The notations $\operatorname{od}(a), i d(a)$, and $d(a)$ are defined as follows: $\operatorname{od}(a)$ is the number of terms in $D$ which start from $a, i d(a)$ is the number of terms in $D$ which end at $a$. and $d(a)=\operatorname{od}(a)+\imath d(a)$ is called the degree of $a$. An even (odd) point is a point with even (odd) degree.

For example, if $D$ is the digraph $D=(a, a) \cdot(a, d) \cdot(c, a) \cdot(b, a) \cdot(b, a)$ then $\operatorname{od}(a)=2, \imath d(a)=4$, $o d(b)=2, i d(b)=0, \operatorname{od}(c)=1, \imath d(c)=0, \operatorname{od}(d)=0, i d(d)=1$, and $\bigwedge D=\emptyset$. The diagram for $D$ can be constructed as:

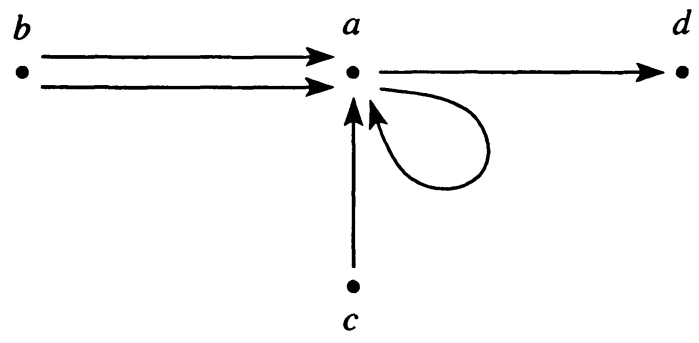

Let $\alpha$ be a permutation of $D$, and define the following functions. Let $\epsilon(\alpha)=1$ if $\alpha$ is an Eulerian path or circuit, and let $\epsilon(\alpha)=0$ if $\alpha$ is not. Let $\pi(\alpha)$ be the sign of the permutation $\alpha ; \pi(\alpha)=+1$ if $\alpha$ is a multiple of an even number of transpositions, and $\pi(\alpha)=-1$ if $\alpha$ is a multiple of an odd number of transpositions. Let $g(\alpha)=\epsilon(\alpha) \cdot \pi(\alpha)$.

What we are looking for is a natural, intuitive proof of the formula:

$$
\sum g(\alpha)=0=\sum_{\alpha \in \Lambda_{x} D} g(\alpha) \text { for any } x \in V D,
$$

if $|V D|=n$ and $|D| \geq 2 n$ for all integers $n \geq 1$.

\section{DISCUSSION AND ARGUMENTS.}

For convenience of notation, we define $g\left(\bigwedge_{x} D\right)=\sum_{\alpha \in \bigwedge_{x} D} g(\alpha)$ and define $g(\bigwedge D)$ similarly. If $\bigwedge_{a} D=\emptyset$, then naturally we take $g\left(\bigwedge_{a} D\right)=0$. For example, if $D=(a, b) \cdot(a, c) \cdot(b, a) \cdot(a, a)$, with diagram

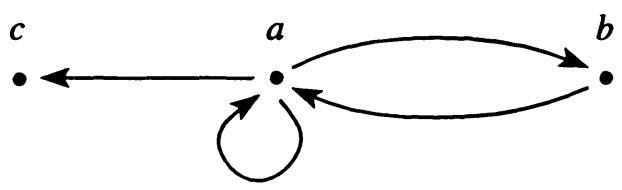

then $\bigwedge_{a} D=\{(a, a) \cdot(a, b) \cdot(b, a) \cdot(a, c),(a, b) \cdot(b, a) \cdot(a, a) \cdot(a, c)\}, \bigwedge_{b} D=\bigwedge_{c} D=\emptyset, g((a, a) \cdot$ $(a, b) \cdot(b, a) \cdot(a, c))=1, g\left(\bigwedge_{a} D\right)=2$, while $g\left(\bigwedge_{b} D\right)=g\left(\bigwedge_{c} D\right)=0$.

For all positive integers $n$, let $B_{n}$ be the family of digraphs $D$ such that

1. $|D| \geq 2 n$ and $|V D|=n$,

2. $i d(x)+o d(x) \geq 3$ for all $x \in V D$;

while $B_{n}^{\prime}$ denotes the subfamily of digraphs $D$ such that

1. $|D|=2 n$ and $|V D|=n$,

2. $i d(x)+o d(x) \geq 3$ for all $x \in V D$. 
For all positive integers $n$, let $A_{n}$ be the family of digraphs $D$ such that

1. $|D| \geq 2 n$ and $|V D|=n$,

2. For some $q \in V D, \imath d(q)+o d(q)=1$ or 2 .

The proposition $S_{n}$ is the following:

$$
\text { "For } j=1, \ldots, n \text {, if } D \in A, \cup B_{\jmath}, \text { then } g(\bigwedge D)=0 . "
$$

In Lemma 3.1 we demonstrate that $S_{1}, S_{2}$, and $S_{3}$ are true. In order to prove $S_{n}$ in general we proceed by induction on $n$, assuming $S_{n-1}$. In computing $g(\wedge D)$, we seek to identify $\wedge D$ with a union of suitable $\bigwedge D_{\imath}^{\prime}$, where $\left|V D_{\imath}^{\prime}\right|<\left|V D_{\imath}\right|$. This is accomplished by identification of a length 3 path with an arc or by deleting some paths in order to maintain the relation that $g(\Lambda D)=0$ if $g\left(\Lambda D_{\imath}^{\prime}\right)=0$ for all $i$.

For instance, as a trivial example, let $(a, v),(v, w),(w, b) \in D$ and $(a, v) \cdot(v, w) \cdot(w, b) \subseteq \alpha$ for all $\alpha \in \bigwedge_{x} D$. Let, when $x \neq v$ and $x \neq w, D^{\prime}=(D-(a, v)-(v, w)-(w, b)) \cdot(a, b)$, and let $F$ be a map of $\Lambda D^{\prime}$ to $\Lambda D$ defined by $F(\alpha)=\beta(a, b) \gamma$ where $\alpha=\beta(a, v) \cdot(v, w) \cdot(w, b) \gamma$. Then $F$ is a one-to-one map and when $\alpha, \beta \in \Lambda D^{\prime}, \beta$ is an even permutation of $\alpha$ if and only if $F(\beta)$ is an even permutation of $F(\alpha)$. Thus $g\left(\Lambda D^{\prime}\right)=0$ implies $g(\Lambda D)=0$.

In particular we assume, based on this observation, that there are no vertices $v$ and $w$ of $V D$ for which there is a path of the type just described, when we assume $S_{n-1}$.

In general, if there are digraphs $D_{1}, D_{2}, \ldots, D_{r}$ and such injective maps $F_{i}$ from $\wedge D_{i}$ to $\wedge D$ such that $\bigcup F_{i}\left(\bigwedge D_{\imath}\right)$ forms a partition of $\Lambda D$ and each $g\left(\Lambda D_{i}\right)=0$, then we can conclude $g(\bigwedge D)=0$.

As another example of an identification of the type described above, if $D$ contains $(a, b)$ and $(b, c)$ and if every $\alpha \in \Lambda D$ starts or ends with $(a, b) \cdot(b, c)$, then take $D^{\prime}=D-(a, b)-(b, c)$. In this case it is also clear that if $g\left(\bigwedge D^{\prime}\right)=0$, then $g(\bigwedge D)=0$.

In all our arguments, it is the case that if a statement is true for a digraph $D$, then it is also true for the digraph obtained by reversing all $\operatorname{arcs}(a, b)$ of $D$ to $\operatorname{arcs}(b, a)$.

If a digraph $D$ contains an arc $(a, b)$ with multiplicity at least two, then trivially $g(\bigwedge D)=0$ since by a simple transposition of one $(a, b)$ with another leaves $D$ unchanged while $g(\bigwedge D)$ changes sign. Therefore we assume no multiple arcs.

Where needed, the truth of the proposition $S_{n-1}$ is assumed in the arguments to follow.

PROPOSITION 1. If there is a vertex $q$ of $D$ with $d(q)=1$ or 2 , then there are digraphs $D_{\imath}$, $i=0,1,2, \ldots$ such that $\left|D_{i}\right| \geq|D|-2,\left|V D_{i}\right| \leq|V D|-1$, and $g(\bigwedge D)=\sum_{i}(-1)^{e_{i}} g\left(\bigwedge D_{i}\right)$, where $e_{i}=0$ or 1 .

PROOF. Suppose $D$ contains arcs $(t, q),(q, h)$, and $\left(h, b_{i}\right), i=1,2,3, \ldots$ Let $D_{z}=(D-$ $\left.(t, q)-(q, h)-\left(h, b_{i}\right)\right) \cdot\left(t, b_{i}\right)$ and $\bigwedge_{i}=\left\{\alpha \in \Lambda D:(t, q) \cdot(q, h) \cdot\left(h, b_{i}\right) \subseteq \alpha\right\} ;$ then we see that $\Lambda D_{i}$ has a one to one correspondence $F_{i}$ as follows: $F_{i}(\alpha)=\alpha_{1}(t, q) \cdot(q, h) \cdot\left(h, b_{i}\right) \alpha_{2}$ if $\alpha=\alpha_{1}\left(t, b_{i}\right) \alpha_{2}$. Clearly $g(\alpha)=(-1)^{e_{1}} g\left(F_{i}(\alpha)\right)$ for some fixed $e_{i}=0$ or 1 , for all $\alpha \in \Lambda D_{i}$. Thus we get $g\left(\bigwedge_{x} D\right)=\sum(-1)^{e_{i}} g\left(\bigwedge_{x} D_{i}\right)$, when $x \neq q$ and $x \neq h$. If $x=h$, or there are no such $b_{i}$, then we can switch $t$ and $h$, and conclude that $g\left(\bigwedge_{x} D\right)=\sum(-1)^{e_{i}} g\left(\bigwedge_{x} D_{\imath}\right)$ if $x \neq q$.

If $d(q)=2$, and $q$ is the starting point, then $\bigwedge_{x} D=\left\{(q, h) \alpha(t, q): \alpha \in \wedge D_{0}\right\}$ where $D_{0}=D-(q, h)-(t, q)$ and it is clear that $g\left(\bigwedge_{x} D\right)= \pm g\left(\bigwedge_{x} D_{0}\right)$.

If $d(q)=1$, then $\bigwedge_{x} D=\left\{(q, h) \alpha: \alpha \in \bigwedge D_{0}\right\}$, where $D_{0}=D-(q, h)$, and it is also clear that $g\left(\bigwedge_{x} D\right)= \pm g\left(\bigwedge_{x} D_{0}\right)$.

Also it is clear that $V D_{i} \not \supset q$ and $\left|D_{i}\right| \geq|D|-2,\left|V D_{i}\right| \leq|V D|-1$. Note that $d(h)$ in $D_{i}$ becomes smaller by 2 except when $h$ is an end point, where it becomes smaller by 1 . We need this fact for the following Lemma 1.1.

LEMMA 1.1. When we assume $S_{n-1}$, the following are true:

(A). If $D \in A_{n}$, then $g(\bigwedge D)=0$. 
(B). If $D \in A_{n+1}$ and there is an arc $(q, h)$ or $(h, q)$ in $D$ such that $d(q)=2$ and $d(h)=3$ or 4 , then $g\left(\bigwedge_{x} D\right)=0$ if $x \neq q$ and $x \neq h$.

PROOF. (A). The digraphs $D_{\imath}$ in Proposition 1 belong to $A_{n-1} \cup B_{n-1}$. Hence $g\left(\bigwedge D_{\imath}\right)=0$ because of $S_{n-1}$, and $g(\bigwedge D)=0$.

(B). In Proposition 1, when $(q, h),\left(h, b_{\imath}\right) \subseteq D$, if $d(h)=3$ or 4 in $D$, then $d(h)$ in $D_{\imath}=1$ or 2, except the case in which $q$ or $h$ is an end point. Thus $D_{\imath} \in A_{n}$ and $g\left(D_{\mathfrak{\imath}}\right)=0$. Thes efore $g\left(\bigwedge_{x} D\right)=\sum(-1)^{e_{1}} g\left(\bigwedge D_{\imath}\right)=0$ if $x \neq q$ and $x \neq h$. When $(h, q) \subseteq D$, we consider $\left(b_{\imath}, h\right) \subseteq D$ instead of $\left(h, b_{\imath}\right)$; then the argument is similar to the case of $(q, h) \subseteq D$.

LEMMA 1.2. When we assume $S_{n-1}$, if a digraph $D \in B_{n}$ has an arc $(t, h)$ such that $t$ and $h$ have degree 3 or 4 , then $g(\bigwedge D)=0$.

PROOF. If $t=h,(t, t) \subseteq D$, or $(h, h) \subseteq D$, then the statement reduces to a case of $S_{n-1}$. So we assume that $t \neq h,(t, t) \notin D$, and $(h, h) \notin D$. According to our assumption, situations illustrated in the following drawings may occur:
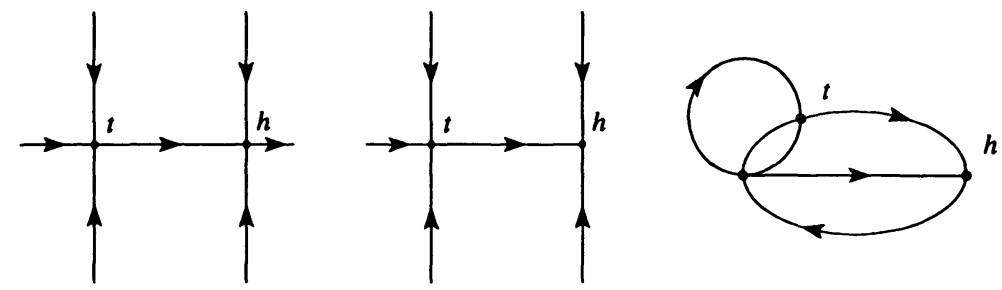

In those diagrams we can interchange $h$ and $t$, and also we can place the arrows in any way so that $\bigwedge D \neq \emptyset$.

Assuming $\bigwedge D \neq \emptyset, d(t)$ and $d(h)=3$ or 4 , we construct a digraph $\bar{D} \in A_{n+1}$ from $D$ as follows.

$\bar{D}=(D-(t, h)) \cdot(t, t) \cdot(t, q) \cdot(q, h)$

$V \bar{D}=V D \cup\{q\}$, as shown in the diagram below.

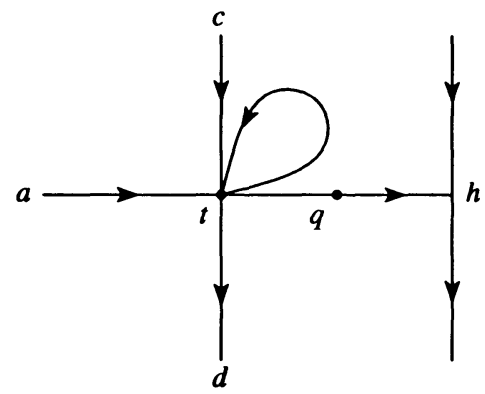

Let $x \in V D$, that is, $x \in V \bar{D}$, and $x \neq q$. From Lemma 1.1.B, $g\left(\bigwedge_{x} \bar{D}\right)=0$ if $x \neq h$. On the other hand, let, for $x \neq t$,

$$
\begin{aligned}
& \bigwedge_{1}=\left\{\alpha \in \bigwedge_{x} \bar{D}:(t, t) \cdot(t, q) \cdot(q, h) \subseteq \alpha\right\} \\
& D_{1}=(\bar{D}-(t, t)-(t, q)-(q, h)) \cdot(t, h) \\
& \bigwedge_{2}=\left\{\alpha \in \bigwedge_{x} \bar{D}:(a, t) \cdot(t, t) \cdot(t, d) \subseteq \alpha\right\} \\
& D_{2}=(\bar{D}-(a, t)-(t, t)-(t, d)-(c, t)-(t, q)-(q, h)) \cdot(a, d) \cdot(c, h) \\
& \bigwedge_{3}=\left\{\alpha \in \bigwedge_{x} \bar{D}:(c, t) \cdot(t, t) \cdot(t, d) \subseteq \alpha\right\} \\
& D_{3}=(\bar{D}-(c, t)-(t, t)-(t, d)-(a, t)-(t, q)-(q, h)) \cdot(c, d) \cdot(a, h)
\end{aligned}
$$

Then $\Lambda_{1}, \Lambda_{2}$, and $\Lambda_{3}$ form a partition of $\Lambda \bar{D}_{x}$, and we see that $g\left(\bigwedge_{1}\right)= \pm g\left(\bigwedge_{x} D_{1}\right)$ by identifying $(t, t) \cdot(t, q) \cdot(q, h)$ to $(t, h)$, we see that $g\left(\bigwedge_{2}\right)= \pm g\left(\bigwedge_{x} D_{2}\right)$ by identifying $(a, t) \cdot(t, t) \cdot(t, d)$ 
to $(a, d)$ and $(c, t) \cdot(t, q) \cdot(q, h)$ to $(c, h)$, and we see that $g\left(\bigwedge_{3}\right)= \pm g\left(\bigwedge_{x} D_{3}\right)$ by identifying $(c, t) \cdot(t, t) \cdot(t, d)$ to $(c, d)$ and $(a, t) \cdot(t, q) \cdot(q, h)$ to $(a, h)$.

If $d(t)=3$ and $(a, t)$ is not an $\operatorname{arc}$ of $D$, then

$$
\begin{aligned}
& \bigwedge_{2}=\left\{\alpha \in \bigwedge_{x} \bar{D}:(t, t) \cdot(t, d) \subseteq \alpha\right\} \\
& D_{2}=(\bar{D}-(t, t)-(t, d)-(c, t)-(t, q)-(q, h)) \cdot(c, h) \\
& \bigwedge_{3}=\left\{\alpha \in \bigwedge_{x} \bar{D}:(c, t) \cdot(t, t) \cdot(t, d) \subseteq \alpha\right\} \\
& D_{3}=(\bar{D}-(c, t)-(t, t)-(t, d)-(t, q)-(q, h)) \cdot(c, d)
\end{aligned}
$$

and the results are the same.

Moreover, it is clear that $g\left(\bigwedge D_{1}\right)=g(\bigwedge D)$. Since $D_{2}$ and $D_{3}$ do not contain vertices $t$ and $q$. $D_{2}, D_{3} \subseteq A_{n-1} \cup B_{n-1}$. Thus $g\left(\bigwedge D_{2}\right)=0$ and $g\left(\bigwedge D_{3}\right)=0$. Together with $g\left(\bigwedge_{x} \bar{D}\right)=0$, we get $g\left(\bigwedge D_{1}\right)=0$, so we get $g\left(\bigwedge D_{x}\right)=0$ for $x \neq h$. In the case $h=x$, we can switch $t$ and $h$. Thus $g\left(\bigwedge_{x} D\right)=0$ for all $x \in V D$, or $g(\bigwedge D)=0$.

PROPOSITION 2. Suppose $D \subseteq B_{n}^{\prime}$ and $\bigwedge D \neq \emptyset$. Also we assume that $n \geq 4$, and that $D$ does not contain any arc of multiplicity more than one, nor any vertex $v$ such that $d(v)=4$ and $\operatorname{arc}(v, v) \subseteq D$. Then $D$ contains an arc $(t, h)$ such that $t \neq h$, one of $t$ and $h$ has degree 4 and the other has degree 4 or 3 .

PROOF. The fact that $D \in B_{n}^{\prime}$ implies that $\sum_{v \in V D} d(v)=4 n$, and $d(v) \geq 4$ for all even points $v$ of $D$. Since $\bigwedge D \neq \varnothing$, the number of odd points is 0 or 2 . If it is 0 , then $d(v)=4$ for all $v \in V D$ : thus our assertion is true. In the case of 2 , say $a$ and $b$ are odd points, and $d(a)+d(b)=6$ or 8 . If $d(a)+d(b)=6$, then there must be $c \in V D$ such that $d(c)=6$ and all other vertices have degree 4 , while $n \geq 4$ implies there is a vertex $d$ beside $a, b$, and $c$. If $d$ incidents only to $c$, then the arc $(c, d)$ or $(d, c)$ has the multiplicity more than 1 . So $d$ must incident to $a$ or $b$ or a point of degree 4 . Thus the assertion is true, because $d(d)=4$ and $d(a)=d(b)=3$.

In the case $d(a)+d(b)=8$, then say $d(a)=3$ and $d(b)=5$. Since $n \geq 4$, there are at least two more vertices, say $c$ and $d$, such that $d(c)=d(d)=4$. If any vertices of degree 4 do not incident to each other and also do not incident to $a$ then all vertices of degree 4 must incident to $b$, thus $d(b) \geq 8$. This contradicts with $d(b)=5$. Thus some vertex of degree 4 has to be incident to the vertex $a$ or a vertex of degree 4 . Thus our assertion is true.

LEMMA 2.1. If $D \in B_{n}^{\prime}, n \geq 4$, and we assume $S_{n-1}$, then $g(\bigwedge D)=0$.

PROOF. From Proposition 2, $D$ has an arc $(t, h), t \neq h$, such that one of $t$ and $h$ has degree 4 and the other has degree 3 or 4 . Then by Lemma $1.2, g(\bigwedge D)=0$.

PROPOSITION 3. If $g\left(\bigwedge D^{\prime}\right)=0$ for all $D^{\prime} \in B_{n}^{\prime}$, then for all $D \in B_{n}, g(\bigwedge D)=0$ when we assume $S_{n-1}$.

PROOF. Let $D \in B_{n}, a \in V D$, and $\alpha \in \wedge D$. Considering a digraph to be a sequence of arcs, it is true that $\bigwedge_{a} \alpha=\bigwedge_{a} D$. Now let $|V D|=n,|D|-2 n=r$ and $\alpha \in \bigwedge_{a} D$. Define $p(\alpha)$ to be the sequence of the first $r$ terms of $\alpha$, while $q(\alpha)$ to be the subsequence of $\alpha$ consisting of the next $2 n$ terms. Thus $\alpha=p(\alpha) q(\alpha)$. Let $\bigwedge_{p(\alpha)}=\left\{\beta \in \bigwedge_{a} D: p(\beta)=p(\alpha)\right\}=\left\{p(\alpha) \xi: \xi \in \bigwedge_{b} q(\alpha)\right\}$, where $b$ is the end point of $p(\alpha)$, and $q(\alpha) \in A_{j} \cup B_{j}$ for some $j<n$ or $q(\alpha) \in A_{n} \cup B_{n}^{\prime}$. If $q(\alpha) \in A_{j} \cup B_{\text {, }}$ for some $j<n$, then $g(\bigwedge q(\alpha))=0$ because of $S_{n-1}$. If $q(\alpha) \in A_{n}$ then $g(\Lambda q(\alpha))=0$ from Lemma 1.1.A. If $q(\alpha) \in B_{n}^{\prime}$ then $g(\bigwedge q(\alpha))=0$ from our hypothesis, $g\left(\bigwedge D^{\prime}\right)=0$ if $D^{\prime} \in B_{n}^{\prime}$. Since $\bigwedge D=\bigcup_{\alpha}\{p(\alpha) \beta: \beta \in \Lambda q(\alpha)\}$ where $\alpha$ runs all elements of $\Lambda D$, and $g(\{p(\alpha) \beta: \beta \in \Lambda q(\alpha)\})=$ $\pm g(\bigwedge q(\alpha))=0$, hence $g(\bigwedge D)=0$.

LEMMA 3.1. $S_{1}, S_{2}$, and $S_{3}$ are true.

PROOF. $S_{1}$ is clearly true. If $D \in B_{2}^{\prime}$, then $D$ is one of the following (with the orientation arbitrary): 

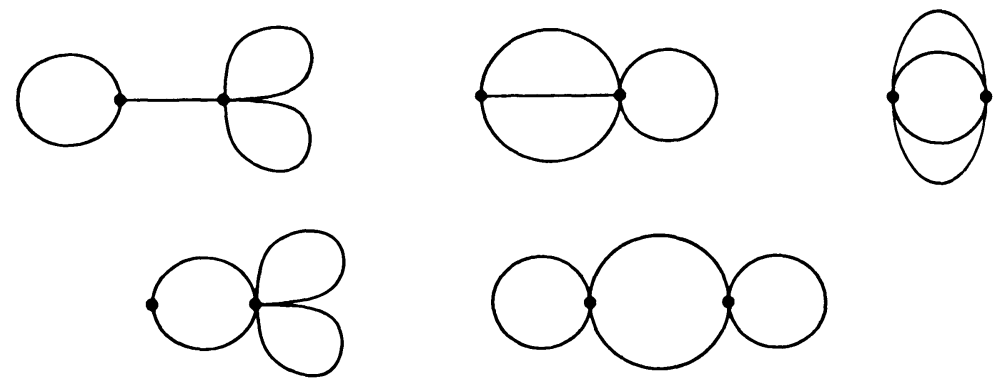

Clearly $g(\bigwedge D)=0$ in all of the above situations. From $S_{1}$ and Proposition 3 , it is true for $B_{2}$ as well as for $A_{2}$ from Lemma 1.1. Thus $S_{2}$ is true.

If $D \in B_{3}^{\prime}$, then $d(v)$ can generate the following sequences when $v$ varies over elements of $I^{\prime} D$ : (A) $(3,3.6),(B)(3,4,5),($ c) $(4,4,4)$. For case (A), it follows that $D$ is one of the following digraphs (with the orientation arbitrary):
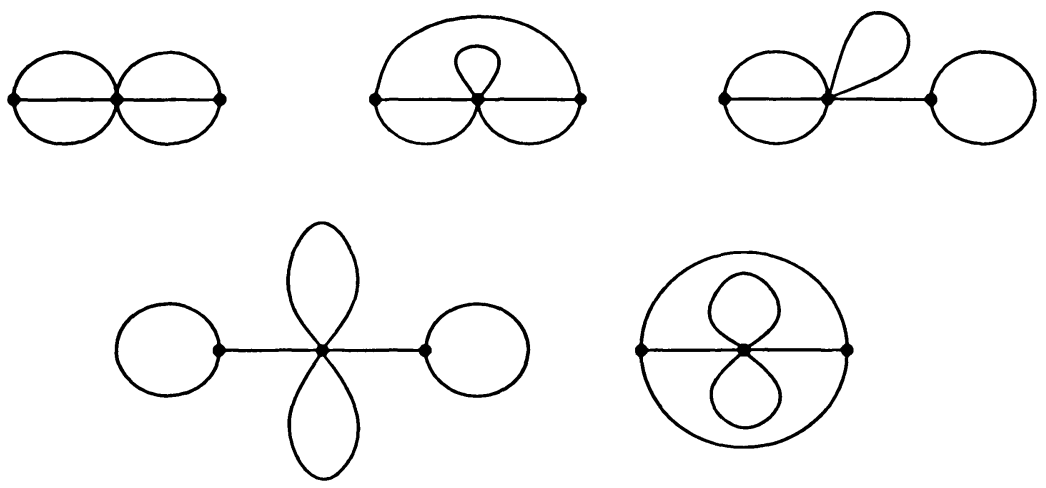

A simple count yields $g(\bigwedge D)=0$ in all cases. In case (B), if there is an arc $(t, h) \subseteq D$ such that $d(t)=4$ and $d(h)=3$, then $g(\bigwedge D)=0$ via $S_{2}$ and Lemma 1.2. Otherwise, it is the case that all Eulerian paths end at the same path of length two or start at the same path of length two. In case (C), $g(\bigwedge D)=0$ as in case (B). Therefore $S_{3}$ follows, by Proposition 3 and Lemma 3.1.

To conclude, we can now claim:

THEOREM. If $D$ is a digraph such that $|V D|=n$ and $|D| \geq 2 n$, then $g(\bigwedge D)=0$; that is, the number of even Eulerian paths equals the number of odd Eulerian paths.

PROOF. $S_{1}, S_{2}$, and $S_{3}$ are true by Lemma 3.1. For $S_{n}$, where $n \geq 4$, we proceed by induction on $n$. When we assume $S_{n-1}$ for $n \geq 4$, if $D \in A_{n}$ then $g(\bigwedge D)=0$ by Lemma 1.1. Also if $D \in B_{n}$, by Lemma 2.1 and Proposition $3, g(\bigwedge D)=0$.

COROLLARY. If $|V D|=n$ and $|D| \geq 2 n+1$, then $g\left(\bigwedge_{(a, b)} D\right)=0$ for $n=1,2, \ldots$ and for all $(a, b) \subseteq D$, where $\bigwedge_{(a, b)} D=\left\{\alpha \in \bigwedge_{a} D: \alpha\right.$ starts with $\left.(a, b)\right\}$

PROOF. As in the proof of Proposition 3, let $p(\alpha)=(a, b)$ while $q(\alpha)$ is the next $2 n$ terms of $\alpha$. Then $g\left(\bigwedge_{(a, b)} D\right)= \pm g\left(\bigwedge_{b} q(\alpha)\right)$. Since $|q(\alpha)| \geq 2 n$ and $|V q(\alpha)| \leq n$, from our theorem $g\left(\bigwedge_{b} q(\alpha)\right)=0$. Thus $g\left(\bigwedge_{(a, b)} D\right)=0$. When all points are even points, all paths are necessarily circuits, and this is Schützenberger's theorem.

\section{REFERENCES}

1. Claude Berge, Graphs and Hypergraphs, Second revised edition, North-Holland, Amsterdam, 1976.

2. R. G. Swan, An application of graph theory to algebra, Proc. Am. Math. Soc. 14 (1964), 367-373. 


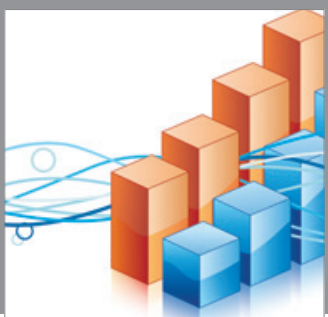

Advances in

Operations Research

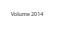

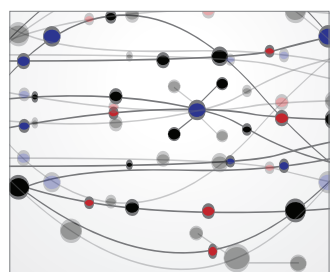

\section{The Scientific} World Journal
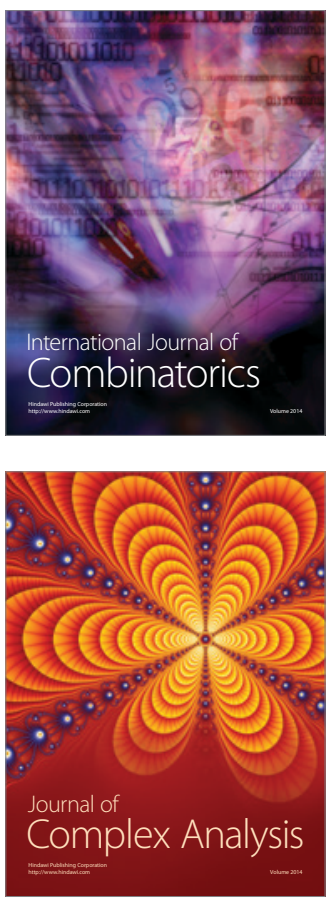

International Journal of

Mathematics and

Mathematical

Sciences
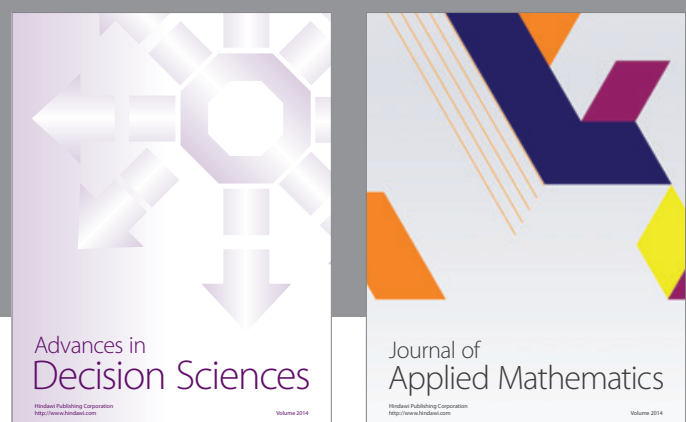

Journal of

Applied Mathematics
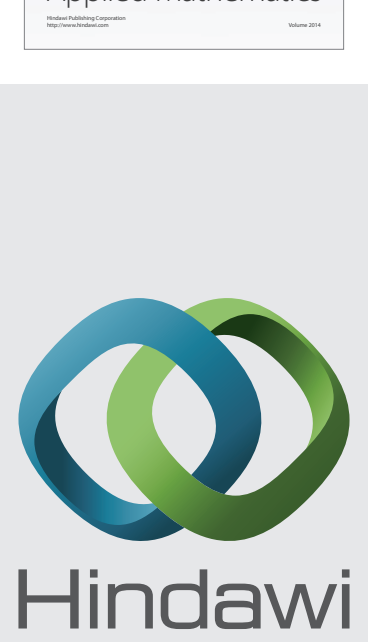

Submit your manuscripts at http://www.hindawi.com
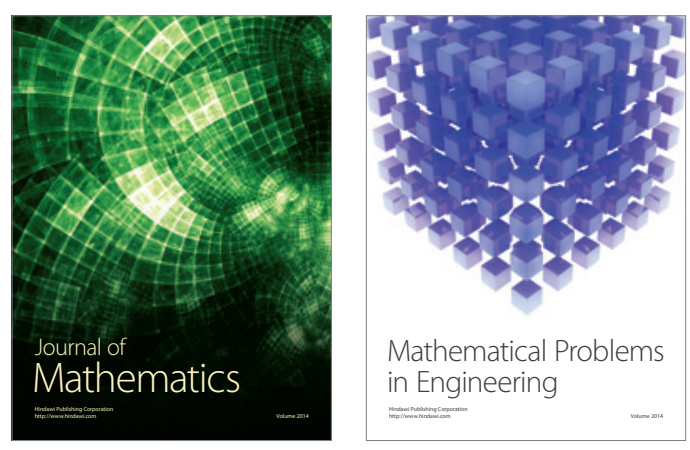

Mathematical Problems in Engineering
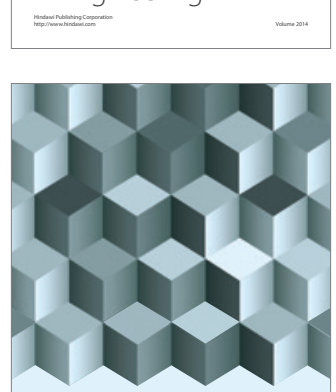

Journal of

Function Spaces
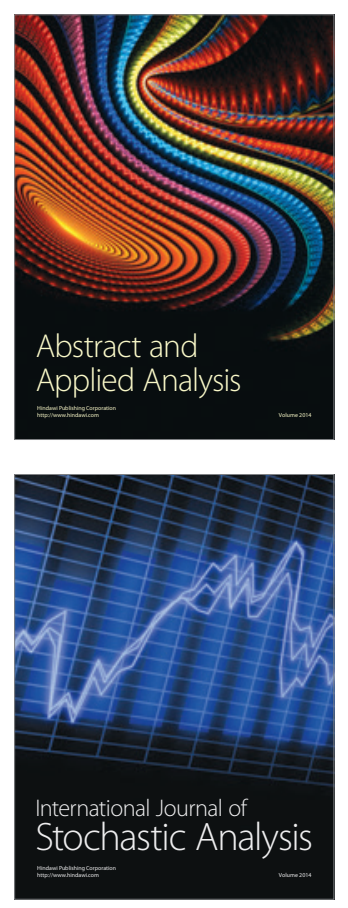

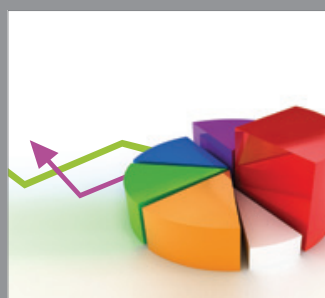

ournal of

Probability and Statistics

Promensencen
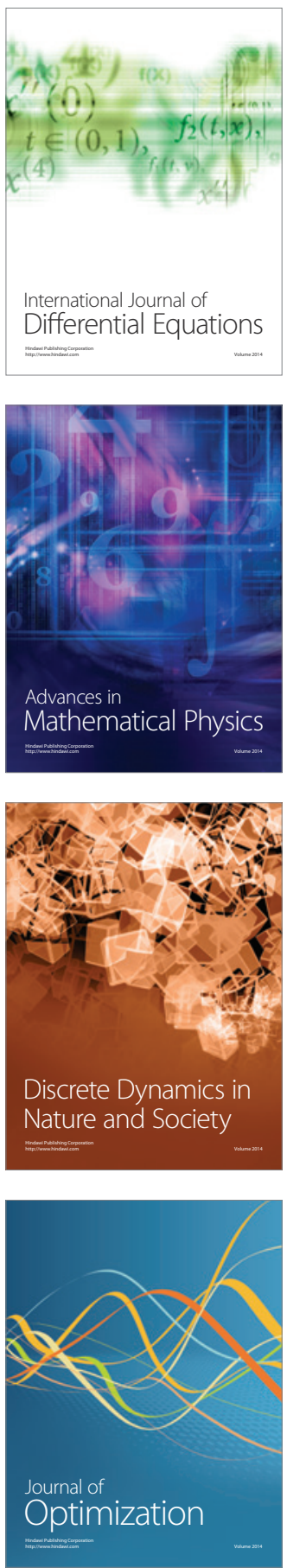